\title{
El conocimiento informático en Ingeniería Industrial. Una visión desde las evaluaciones de la CONEAU
}

Alejandro Héctor Molina ${ }^{1}$

\section{Resumen}

La necesidad del manejo de las herramientas informáticas en las ingenierías, requiere de mayores y mejores niveles de desarrollo de competencias informáticas, tal como propone el documento del CONFEDI sobre competencias genéricas del Ingeniero. Una de las carreras en las cuales la utilización de esas herramientas se hace particularmente sensible, es la Ingeniería Industrial, tanto por la evolución de los sistemas de Información en áreas como Logística, Modelización, Estrategia y Calidad y de los sistemas informatizados para control de procesos. La forma en que se cubre esta necesidad es analizada en función de evaluaciones que realizó la CONEAU en carreras de Ingeniería Industrial en Argentina en el Período 19922012. También se contrasta los contenidos de esos planes, respecto de los contenidos de materias pertenecientes a carrereas específicas de Sistemas y Computación en Argentina y finalmente, respecto de distintos planes de universidades de EEUU.

Palabras Clave: informática, competencias, TIC, acreditación, ingeniería. 


\section{Abstract}

The need for management of IT tools in engineering requires a greater and better computer skills development, as proposed by CONFEDI paper on Engineer generic skills. One of the careers in which the use of such tools is particularly sensitive is Industrial Engineering, due to information system evolution in areas such as Logistics, Modeling, Strategy and Quality and computerized systems for process control. The way to meet this need is analyzed in terms of evaluations conducted by the CONEAU on Industrial Engineering careers in Argentina during 1992-2012. The contents of these plans are contrasted regarding subjects specific to Systems and Computing in Argentina and finally, for different U.S. universities plans.

Keywords: IT, skills, ICT, accreditation, engineering. 


\section{Introducción. La alfabetización informática como esencia del conocimiento informático}

El término alfabetización se interpreta de distintas maneras. En inglés, se utiliza la palabra literacy, para expresar el dominio de una serie de competencias y habilidades de otros campos, utilizándose por ejemplo, media literacy, digital literacy, information literacy o technological literacy, como forma de referirse a estas nuevas "alfabetizaciones" necesarias en la sociedad de la información. Estas nuevas alfabetizaciones relacionadas con el uso de la información lo son para la nueva sociedad, una red de la actualidad. Su objetivo, su para qué, es lograr que los individuos, a través de un proceso de aprendizaje a lo largo de la vida, sean capaces de encontrar, evaluar y usar información de cualquier fuente que, de manera eficaz les permita resolver sus problemas, construir conocimiento y tomar decisiones, además de dominar las herramientas que auxilian las tareas específicas de cada profesional.

En los comienzos, el proceso de alfabetización se orientó al uso de bibliotecas y sus recursos, en los años 50 la biblioteca era el eje alrededor del cual giraban todas las actividades de la universidad (Wilson y Tauber, 1956; Shores, 1970). Posteriormente surge la necesidad de introducir a los estudiantes en la biblioteca como parte integral de la curricula universitaria (Knapp, 1956; Knapp, 1958). En los 80, se distingue entre instrucción en el uso de la biblioteca y la instrucción bibliográfica (Association of College and Research Libraries. Information Literacy And Acrl, 2002 ); la educación de usuarios toma auge, insertándose en la currìcula cursos sobre fuentes de información o técnica bibliográfica (Fjalbrant y Malley, 1984). Esa orientación fue criticada porque el estudiante no tenía que resolver problema alguno, y no se da el aprendizaje. Surge el concepto de alfabetización en información (ALFIN) en un artículo de Zurkowski quien definió: “... los que están adiestrados en la aplicación de recursos de información a su trabajo puede decirse que están alfabetizados en información” (Zurkowski, 1974). Burchinal completa esta idea: "Para ser un alfabeto en información se requieren una serie de nuevas habilidades que incluyen cómo localizar y usar información para la solución de problemas y la toma de decisiones de manera eficiente y efectiva" (Burchinal, 1976).

Por ello se acepta que la capacidad para usar la información tiene relación con el éxito en los estudios, mediante un acercamiento a esta, partiendo de palabras clave, descriptores o términos de búsqueda. Bernhard señala que dichos términos se agrupan en dominios relativos al uso de la información y a aquellos asociados (Bernhard, 2002). En el primero se incluyen los siguientes: uso de la información, competencia en el uso de la información, alfabetización en información, cultura informacional, cultura de la información, alfabetización en el uso de la biblioteca, el proceso de búsqueda de información, la búsqueda documental, recuperación de información, metodología documental. Asimismo comprende la formación en el uso de la 
información, formación documental e instrucción bibliográfica. En el segundo grupo aparecen los que se indican a continuación: formación en tecnologías, competencia en el uso de las tecnologías, alfabetización en el uso de redes informáticas, competencia informática, alfabetización en el uso de medios, educación visual, pensamiento crítico, pensamiento conceptual, métodos del trabajo intelectual, métodos de estudio y, habilidades para el estudio. Esta metodología puede encontrarse en los trabajos de Behrens, Bober, Johnson, O'Sullivan, Rader y Ridgeway (Behrens, 1994; Bober et al, 1995; Johnson, 2001; O'Sullivan, 2002; Rader, 2000; Ridgeway, 1991).

La necesidad de utilizar no solo información, sino las tecnologías asociadas y establecer relaciones de las capacidades de uso de la tecnología y la información sugirió en la visión de Shapiro y Hughes sobre alfabetización tecnológica e informacional y proponen un programa de alfabetización informática basado en siete dimensiones que dan lugar al desarrollo de otras competencias: uso de herramientas informáticas, uso de recursos de información, desarrollo de aptitudes socio-culturales, desarrollo de capacidad investigadora; uso de medios de comunicación, uso de las innovaciones tecnológicas (Association of College and Research Libraries, 1999). Paralelamente, algunos autores destacan la existencia de una brecha digital, por la desigualdad en el acceso a las tecnologías de la información y de las comunicaciones (TIC) que dificulta la ALFIN en las comunidades y países poco desarrollados.

En cuanto a las competencias que se incluyen en la ALFIN, existen muchos modelos con similitudes y diferencias, como son los propuestos por: el ACRL (Association of College and Research Libraries, , 2000a; Association for College and Research Libraries, 2000b; Association for College and Research Libraries, 2001). También se desarrollaron indicadores de evaluación de las distintas competencias (Association for College and Research Libraries, 2002). Estas competencias han sido compiladas (Bernhard, 2000) en las siguientes categorías: Identificación de la necesidad de información, Creación y organización de la información, Estrategias de búsqueda de información, Habilidades tecnológicas e informáticas, Evaluación y tratamiento de la información, Utilización y comunicación de la información, Aspectos éticos y sociales, Actitud activa de cara al aprendizaje para toda la vida, Crítica a los medios de comunicación y Autoevaluación (Bernhard, 2000).

\section{La Educación Superior en la Sociedad de la Información}

En las universidades, las TIC se han extendiendo por toda la comunidad y se han integrado a la mayoría de las actividades académicas y extra-académicas. Sin embargo, suele confundirse la mera alfabetización tecnológica instrumental con la capacidad de evaluación y aplicación de la información más allá de las estrategias de transcripción de textos u otras actividades. La Declaración Mundial sobre la Educación Superior de 
UNESCO (1998) indicó que era necesario un nuevo modelo de educación superior centrado en el estudiante, al que se le debe formar con espíritu crítico para analizar los problemas de la sociedad, buscar soluciones, aplicar éstas y asumir responsabilidades sociales. Asimismo, plantea el surgimiento de una de las piezas clave del siglo XXI: el aprendizaje a lo largo de toda la vida que va más allá de una educación tradicional, inicial o continúa. En el mismo sentido, la Organización para la Cooperación y Desarrollo Económicos (Organización para la Cooperación y Desarrollo Económicos: OECD, 1996), señala que la economía basada en el conocimiento se caracteriza por la continua necesidad de aprender tanto información codificada como las competencias para usarla, sin embargo, como el acceso a la información se facilita cada vez más, a un menor costo, las habilidades y las competencias relacionadas con la selección y uso eficiente de la información se convierten en cruciales.

\section{Objetivos de la Educación Superior en la Sociedad de la Información}

La ALFIN supone la adquisición consecutiva de competencias relacionadas con el proceso de documentarse y de producir nueva información. Una persona que está apta en el acceso y uso de la información, que es capaz de reconocer cuándo necesita información y tiene la habilidad para localizarla, evaluarla y utilizarla eficazmente, domina las siguientes competencias:

- Es capaz de determinar la naturaleza y nivel de la necesidad de información.

- Accede a la información requerida de manera eficiente y eficaz

- Evalúa la información y sus fuentes de forma crítica e incorpora la información seleccionada en el propio cuerpo de conocimientos y el sistema personal de valores.

- Utiliza la información eficazmente para cumplir un propósito específico, individualmente o como miembro de un grupo.

- Comprende muchos de los problemas y cuestiones económicas, legales y sociales que se relacionan con el uso de la información, y accede y utiliza la información de forma ética y legal.

\section{Las TIC en las carreras de ingeniería en Argentina}

El consejo federal de Decanos de Ingeniería (CONFEDI) produjo en 1996 la publicación titulada "Unificación Curricular en la Enseñanza de las Ingenierías en la República Argentina", conocido como "Libro Azul”, en 2000, generó la "Propuesta de Acreditación de Carreras de Grado de Ingeniería en la República Argentina", llamado "Libro Verde". Estos fueron la base de las posteriores Resoluciones del Ministerio de Educación, Ciencia y Tecnología, para establecer los estándares en la 
enseñanza de la ingeniería en la Argentina. En 2001, la CONEAU los utilizó como base para los procesos de acreditación de carreras de ingeniería en Argentina. En 2004, el CONFEDI propuso un "Proyecto Estratégico de Reforma Curricular de las Ingenierías", base de varios documentos,, entre ellos el llamado "Documento de Santa Fe" (2007), donde se avanzó en áreas generales, pero no se varió sustancialmente la propuesta original del "Libro Azul" de 1996, en lo referente a las propuestas curriculares que constituyen el eje de la alfabetización informática.

Este punto es crucial, para entender cómo se piensa la enseñanza de los temas relacionados con el proceso de alfabetización informática en las carreras de ingeniería, ya que mediante el proceso de acreditación de las carreras de ingeniería realizado por la CONEAU, siguiendo las resoluciones del ministerio de Educación: RM No 1232/01, para Ingeniero Aeronáutico, Ingeniero en Alimentos, Ingeniero Ambiental, Ingeniero Civil, Ingeniero Electricista, Ingeniero Electromecánico, Ingeniero Electrónico, Ingeniero en Materiales, Ingeniero Mecánico, Ingeniero en Minas, Ingeniero Nuclear, Ingeniero en Petróleo, Ingeniero Químico; RM N ${ }^{\circ} 1054 / 02$, para Ingeniero Industrial e Ingeniero Agrimensor; y RM N ${ }^{\circ} 013 / 03$, para Ingeniero Hidráulico e Ingeniero en Recursos Hídricos, se establecen las pautas del diseño curricular de las materias de la distintas carreras de ingeniería. Cuyo detalle puede observarse en la tabla 1

TABLA 1. Carga Horaria de los ejes de Formación General y Básica

\begin{tabular}{|c|c|c|c|c|}
\hline Eje de Formación & Área del Conocimiento & Sub área del Conocimiento & Horas & Horas \\
\hline \multirow{5}{*}{\multicolumn{2}{|c|}{$\begin{array}{l}\text { Formación General } \\
\text { Subtotales mínimos del eje } \\
150 \text { horas } \\
8,5 \text { créditos }\end{array}$}} & Lenguas no maternas & \multicolumn{2}{|c|}{ Extracurricular } \\
\hline & & Comunicación Oral y Escrita & 25 & 2 \\
\hline & & Ética & 25 & 1,5 \\
\hline & & Sistemas de Representación & 50 & 2,5 \\
\hline & & Informática & 50 & 2,5 \\
\hline \multirow{9}{*}{$\begin{array}{l}\text { Formación Básica } \\
\text { Subtotales míni- } \\
\text { mos del eje } \\
675 \text { horas } \\
53,5 \text { créditos }\end{array}$} & \multirow{3}{*}{$\begin{array}{l}\text { Ciencias Básicas } \\
\text { Comunes } \\
\text { Subtotales del Área } \\
600 \text { horas } \\
48 \text { créditos }\end{array}$} & Matemática & 350 & 28 \\
\hline & & Estadística & 50 & 4 \\
\hline & & Física & 200 & 16 \\
\hline & \multirow{5}{*}{$\begin{array}{l}\text { Ciencias Básicas Espe- } \\
\text { cíficas } \\
\text { Subtotales del Área } \\
50 \text { horas } \\
3,5 \text { créditos }\end{array}$} & Química & \multirow{5}{*}{50} & \multirow{5}{*}{3,5} \\
\hline & & Geología & & \\
\hline & & Matemáticas Especiales & & \\
\hline & & Ecología & & \\
\hline & & Biología & & \\
\hline & $\begin{array}{l}\text { Ciencias Sociales } \\
\text { Subtotales del Área } \\
25 \text { horas } \\
2 \text { créditos }\end{array}$ & $\begin{array}{l}\text { Fundamentos de Economía, } \\
\text { Política y Sociología }\end{array}$ & 25 & 2 \\
\hline
\end{tabular}

Fuente CONFEDI 5. 


\section{Análisis de la curricula de informática en carreras de ingeniería.}

En el año 1996 se sentaron las bases para determinar la carga horaria de las materias, una primera aproximación puede hacerse desde dos puntos de vista: el primero desde el análisis de la carga horaria relativa de las materias de informática y el segundo desde los contenidos de dichas materias.

Analizando la carga horaria de las materias de informática, vemos que esta se establece en 50 horas para informática afrente a las 825 horas del total correspondiente a las materias de formación básicas y general, siendo su carga horaria un $6 \%$ de la carga total del área general y básica. Suele integrarse con informática las materias de sistemas de representación, algo que podría objetarse, pero aún así, ambas constituyen el 12\% de la carga horaria total. La CONEAU, integra en las resoluciones antes mencionadas ambas áreas en el apartado 4.

Respecto de los contenidos, debe indicarse que desde 1996 se han producido cambios notables, un ejemplo de ello es la evolución que han tenido los sistemas de información durante los últimos 20 años, como puede verse en el Diagrama 1. Desde 1996, donde se fijan estos contenidos se han desarrollado 5 nuevas versiones del Sistemas Operativo más frecuentemente utilizado (de la versión 95 del Sistema Operativo Windows, hasta la versión 7), se han desarrollado 4 nuevas versiones de la suite de utilidades Office (desde la versión 95 hasta la versión 2010), el programa Autocad, de la firma Autodesk, ha presentado 5 nuevas versiones y la mayoría de los programas que constituyen la base del software más frecuentemente utilizado, han realizado cambios significativos y presentado no menos de 4 nuevas versiones. Los procesadores que se utilizaban en 1996 han aumentado su velocidad en más de 10 veces, la capacidad de almacenamiento de información se ha multiplicado por 100, la velocidad de comunicación de redes se multiplicó por 10 y la velocidad de acceso a internet en 50 veces. En síntesis, la velocidad de innovación que tienen las TIC obliga a generar mecanismos curriculares que consideren las importancia relativa que están han adquirido recientemente y que alteran el horizonte de la alfabetización informática provista en las carreras de ingeniería.

DIAGRAMA 1. Evolución de los Sistemas de Información

Líneas Generales de Evolución
OLTP: On Line Transactional Systems
OLAP: On Line Analytical Process
Elementos que componen las líneas generales
TPS: Transactional Process Systems
MIS: Management Information Systems
DSS: Decision Support Systems
EIS: Executive Information Systems
OAS: Office Automation Systems
SE: Experts Systems
ERP: Enterprise Resource Planning
BI: Business Intelligence


Adicionalmente los temas que se incluyen para tratarse en materias de informática, como se aprecia en la Tabla II, alternan sin coherencia aspectos básicos de los conocimientos informáticos con aspectos relativos a programas de aplicación, sin considerar que para muchos programas de aplicación es necesario contar con conocimientos básicos que hacen a los requerimientos de una verdadera alfabetización informática que permita mantener un nivel creciente de aptitudes frente a las constantes innovaciones del sector de las Tics.

\section{TABLA II. Contenidos Temáticos de las áreas de Informática}

\begin{tabular}{|c|c|c|}
\hline Informática & $\begin{array}{l}\text { Manejar recursos informáticos } \\
\text { (interfaz gráfica, procesador de } \\
\text { textos, planilla de cálculo, base de } \\
\text { datos, servicios de internet) como } \\
\text { herramientas auxiliares para la } \\
\text { resolución de problemas especí- } \\
\text { ficos. } \\
\text { Elaborar estrategias lógicas (pro- } \\
\text { gramas) para resolver problemas. }\end{array}$ & $\begin{array}{l}\text { Elementos fundamentales de la } \\
\text { interface gráfica: ventanas, íconos, } \\
\text { uso de menús, herramientas. } \\
\text { Procesador de textos. } \\
\text { Planilla de cálculo. } \\
\text { Base de datos. } \\
\text { Servicios de internet. } \\
\text { Técnicas de programación. } \\
\text { Lenguaje de programación. }\end{array}$ \\
\hline $\begin{array}{l}\text { Sistemas de } \\
\text { representación }\end{array}$ & $\begin{array}{l}\text { Representar y visualizar objetos } \\
\text { mediante el uso de sistemas de } \\
\text { representación gráfica manual. } \\
\text { Utilizar software básico de diseño } \\
\text { asistido por computadora para } \\
\text { solucionar problemas simples. }\end{array}$ & $\begin{array}{l}\text { Vocabulario técnico de sistemas } \\
\text { de representación. } \\
\text { Normas. } \\
\text { Sistemas de representación. } \\
\text { Proyecciones geométricas. } \\
\text { Vistas. } \\
\text { Normas de acotación. } \\
\text { Escalas. } \\
\text { Perspectivas. } \\
\text { Técnicas de visualización. } \\
\text { Programas de diseño asistido por } \\
\text { computadora. }\end{array}$ \\
\hline
\end{tabular}

Fuente: CONFEDI

Al hacer un análisis conjunto de los anteriores aspectos: tiempo y contenidos, vemos que la introducción de elementos de informática en materias más avanzadas de la carrera, genera una brecha de conocimientos, que lleva a una subutilización de las herramientas informáticas disponibles dejando al fututo ingeniero como usuario medio de herramientas fundamentales en la aplicación de sus conocimientos.

\section{Análisis de carreras de Ingeniería Industrial evaluadas por la CONEAU}

El análisis de la carrera de Ingeniería Industrial, presenta es un caso ilustrativo del análisis anterior. En particular las competencias que se establecen en forma genérica para los ingenieros industriales requieren de una profunda alfabetización 
informática en los términos que se indico anteriormente en el punto 1. En particular la informática acompaña como herramienta fundamental más del $50 \%$ de las competencias de un ingeniero industrial. Para el actual estudio se analizaron los casos de 31 carreras de Ingeniería Industrial acreditadas por la CONEAU, de modo de tener una muestra que cumplía los estándares de las Resolución Ministerial $\mathrm{N}^{\mathrm{o}}$ 1054/02, que requiere una horaria mostrada en la Tabla III

TABLA III. Cargas Horarias Mínimas

\begin{tabular}{|l|l|}
\hline \multicolumn{1}{|c|}{ Ciencias Básicas } & \multicolumn{1}{|c|}{$\begin{array}{c}\text { Carga horaria mínima según } \\
\text { Resolución 1054/02 }\end{array}$} \\
\hline Matemática & 400 horas \\
\hline Física & 225 horas \\
\hline Química & 50 horas \\
\hline Sist. Representación y Fund. de Informática & 75 horas \\
\hline Total & 750 horas \\
\hline
\end{tabular}

Fuente; CONEAU

Se verificó que ninguna de las carreras acreditadas, contenía una carga menor a la propuesta por el CONFEDI (Tabla I), que resulta en 25 horas más de las requeridas en la Resolución 1054/02 (Tabla III). Se realizó un análisis de corte transversal con la carga horaria de las materias de informática en relación a la carga horaria total, su resultado puede apreciarse en el Grafico 1

GRAFICO 1. Cantidad porcentual de carga horaria relativa a la carga horaria tota

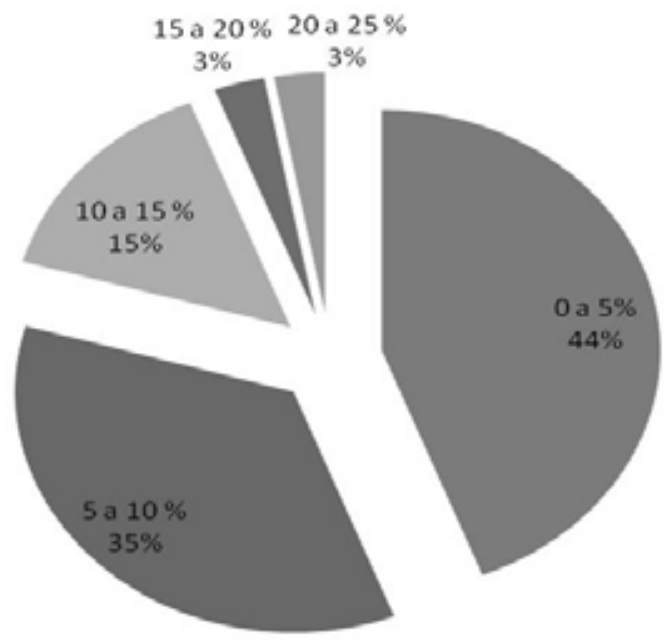


Respecto de los contenidos, se analizó la dedicación promedio estimada, de las áreas generales de conocimientos, la que se resume en el Gráfico 2,

GRAFICO 2. Distribución porcentual de la carga horaria según distintas áreas, en las carreras acreditadas por la CONEAU

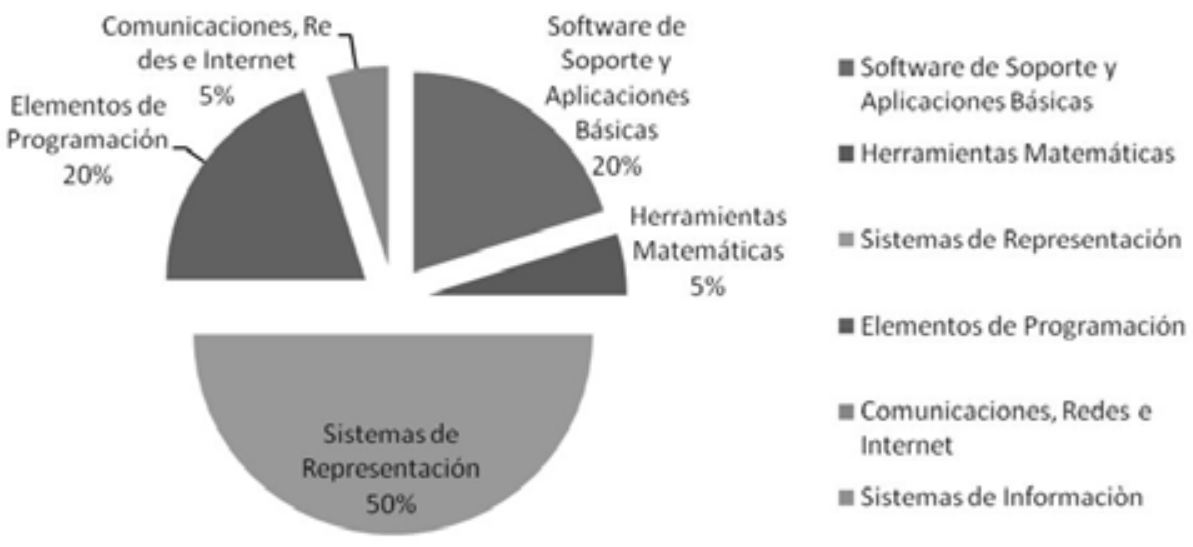

A efectos de establecer un parámetro comparativo, se utilizó una combinación entre los contenidos de las carreras de informática como Licenciaturas en Ciencias de la Computación e Ingeniería en Sistemas y carreras de Ingeniería de EEUU como el MIT y el CALTECH, el resultado ponderado se resume en el gráfico 3

\section{GRAFICO 3. Distribución ponderada de los porcentajes de la carga} horaria, según carreras de informática y universidades de EEUU

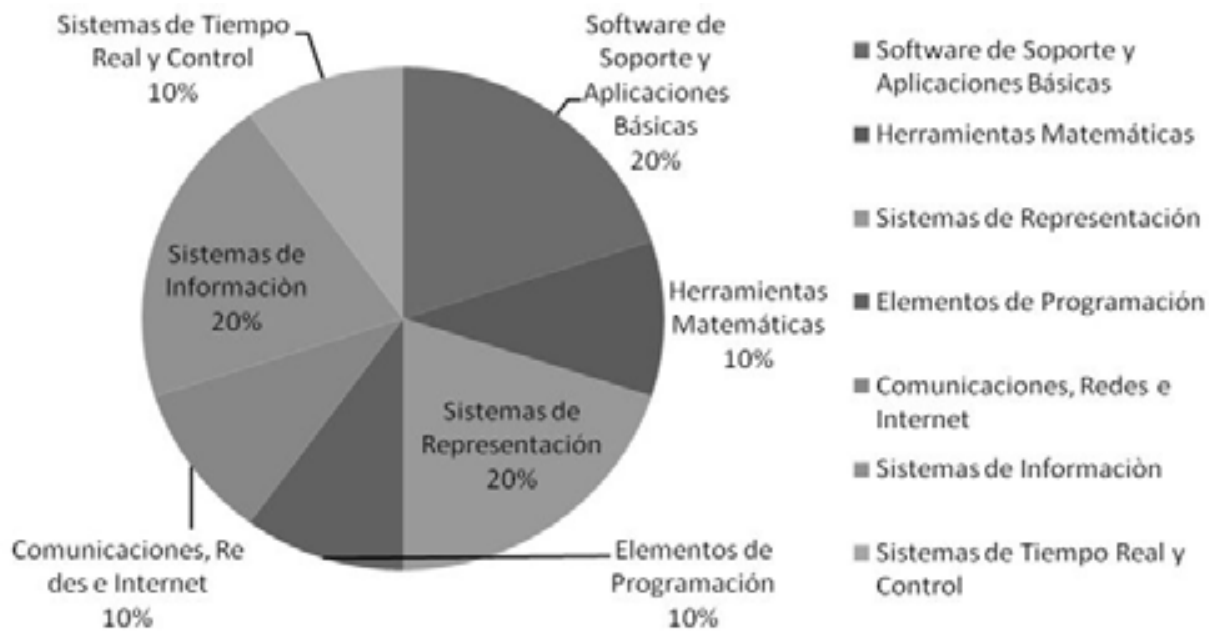




\section{Conclusiones}

El conocimiento informático implica el desarrollo de habilidades básicas que permitan no solo la autoeducación continua del futuro ingeniero sino destrezas que exceden el marco de las competencias específicas del ingeniero, pero que son esenciales para que este cumpla su rol la sociedad de la información en que vivimos, hoy esa competencia debe adquirirla fuera de la currícula universitaria, con el consabido costo individual o social. En este contexto, la formación de los ingenieros para lograr este proceso no cuenta con los elementos de formación básica que aseguren una adecuada alfabetización informática en los términos en que esta se requiere, dada la velocidad de la innovación que tienen las Tics, el caso de la carrera de Ingeniería Industrial, analizada es una prueba de ello. Por ello es necesario replantear la enseñanza de la informática en las carreras de ingeniería, no como complemento práctico de materias de especialización técnica, sino desde la formación de aptitudes que permitan la evolución del conocimiento mediante la aplicación del mismo en las materias de especialización y la actualización del mismo la ritmo que impone el desarrollo de la tecnología de la información.

\section{Bibliografía}

ASSOCIATION OF COLLEGE AND RESEARCH LIBRARIES (1999), Normas y directrices de la ACRL/ALA sobre servicios bibliotecarios en universidades e instituciones de educación superior, III. Normas para bibliotecas de centros universitarios de pregrado. Disponible: http://www.aab.es/033trad.htm.

ASSOCIATION OF COLLEGE AND RESEARCH LIBRARIES.(2000A). "Information Literacy Competency Standards For Higher Education". Disponible: http://www.ala.org/acrl/ilintro.html.

ASSOCIATION FOR COLLEGE AND RESEARCH LIBRARIES.(2000B). "Information Literacy Competency Standards For Higher Education: Standards, Performance Indicators, and Outcome's". Disponible en: htt.:/www.ala.org/acrl/ ilstandardlo.html.

ASSOCIATION FOR COLLEGE AND RESEARCH LIBRARIES (2000C). "Normas Sobre Aptitudes Para el Acceso y uso de la Información en la Enseñanza Superior". Disponible en: http://www.ala.org/acrl/ilintro_span.html. 
ASSOCIATION OF COLLEGE AND RESEARCH LIBRARIES. (2001). Objectives For Information Literacy Instruction: A Model Statement For Academic Librarians. Disponible en : http://www. ala.org/acrl/guides/objinfolit.html, 2001.

ASSOCIATIONOF COLLEGEANDRESEARCHLIBRARIES.(2002). Information Literacy And Acrl. Disponible en : http://www.ala.org.acrl/1//acrl/report.htm.

BEHRENS, S. (1994). Conceptual Analysis and Historical Overview Of Information 15 Literacy. College And Research Libraries, Vol. 55, 309-322.

BERNHARD, P. (1998) Aprendre À Maîtriser L'information: Des Habilités Indispensables Dans Une Societé Du Savoir. Education et Francophonie, Disponible: http://mapageweb.umontreal.ca/bernh/aaafd.97/aafd.html.

BERNHARD, P. (2002). "La Formación En El Uso De La Información: Una Ventaja En La Enseñanza Superior. Situación Actual”. Anales de Documentación, Vol 13.

BOBER C., POULIN, S Y VILENO, L. (1995). "Evaluating Library Instruction In Academic Libraries: A Critical Review Of The Literature, 1980-1993". Reference Librarian Vol.15.

BRETELLE-DESMAZIERES, D. (1998). Aperçu Des Caractérostoqies Des Formations À L'usage De L'information Dans L'enseignement Supérieur Français. Education Et Francophopnie. Disponible en: http://www.acelf.ca/revue/xxvi-1/ articles/11-bretelle.html.

BURCHINAL, L. (1976). "The Future Of Organizing Knowledge”. Centennial Academic Assembly, Texas A\&M University.

CONEAU (1999) Ordenanzas No 005-Coneau-99.

CONEAU (2002) Ordenanzas No 032-Coneau-02.

CONEAU (2004) Resolución No 028/04.

CONEAU (2004) Resolución No 123/04.

CONEAU (2005) Resolución No 071/05.

CONEAU (2005) Resolución No 072/05.

CONEAU (2005) Resolución No 412/05.

CONEAU, (2002-2012) Resoluciones Sobre Acreditación De Carreras De Ingeniería Industrial

CONFEDI (1996) "Unificación Curricular en la Enseñanza de las Ingenierías en la República Argentina”, Libro Azul. 
CONFEDI (2000) "Propuesta de Acreditación de Carreras de Grado de Ingeniería en la República Argentina", Libro Verde.

CONFEDI (2007) "Proyecto Estratégico De Reforma Curricular De Las Ingenierías 2005 - 2007”, Documento De Santa Fe.

COUNCIL OF AUSTRALIAN UNIVERSITY LIBRARARIANS. (2001) "Information Literacy Standards", Ed. Canberra

FJALBRANT, N. Y MALLEY, I. (1984) “User Education In Libraries”.(2nd Ed.) Ed.: Clive Bingley. London.

GILSTER, P. (1997), "Digital Literacy”, Ed. Wiley. New York, 1997.

GÓMEZ HERNÁNDEZ, J. (2000), "La Alfabetización Informacional y la Biblioteca Universitaria. Organización de Programas para Enseñar el Acceso y Uso de la Información". Ed. Murcia.

GRASSIAN, E. Y KAPLOWITZ, J (2001) "Information Literacy Instruction". Ed. Neal-Schuman Publishers, New York,

JOHNSON, A., (2001) "Library Instruction and Information Literacy - 2000w". Reference Services Review Vol. 19.

KNAPP, P. (1956) "A Suggested Program Of College Instruction In The Use Of The Library" Library Quarterly.

KNAPP, P. (1958) “College Teaching And The Library”. Ed. Illinois Libraries.

LEY NACIONAL No 24.521 y Decretos Reglamentarios No 173/96, No $705 / 97$ Y $\mathrm{N}^{\circ}$ 499/95, (1994)

MCMAHON, C. Y BRUCE, C. (2002), "Information Literacy Needs Of Local Staff In Crosscultural Development Projects". Journal Of International Development, VOL. 14.

MINISTERIO DE EDUCACIÓN CIENCIA Y TÉCNICA, Resolución MECYT $\mathrm{N}^{\mathrm{o}} 1054 / 02$,

OBERMAN, C. (2002) "What The Acrl Institute For Information Literacy Best Practices Initiative Tells Us About The Librarian As A Teacher". Ifla. General Conference, Glasgow.

OKER-BLOM, T. (1998), "Integration Of Information Skills In Problem -Based Curricula”. Ifla General Conference.

ORGANIZACIÓN PARA LA COOPERACIÓN Y DESARROLLO ECONÓMICOS. (1996) “The Knowledgebased Economy”. Paris: OECD; 1996. 
O'SULLIVAN, C. (2002),'Is Information Literacy Relevant In The Real World?" Reference Services Review, Vol.21.

RADER, H. (1999).” La Colaboración Entre El Personal Docente E Investigador Y Los Bibliotecarios A La Hora De Elaborar Planes De Estudio Para El Próximo Milenio. La Experiencia En Los Estados Unidos”. 64t H Ifla General Conference.

STEIN, L. Y LAMB, J. (1998), "Not Just Another Bi: Faculty-Librarian Collaboration To Guide Students Through The Research Process". Research Strategies, Vol. 16.

RADER, H. B. (2000). "A SILVER ANNIVERSARY: 25 YEARS OF REVIEWING THE LITERATURE RELATED TO USER INSTRUCTION". Reference Services Review.

RIDGEWAY, T. (1991), "Information Literacy: An Introductory List". Disponible en: www.educause.edu/pub/er/review/reviewarticles/31231.html.

SHORES, L. (1970). "Library-College Usa: Essays On A Prototype For An American Higher Education”. Tallahassee, South Pass Press. pp. 159.

UNESCO. (1996). "Education: The Necessary Utopia: [The Delors Report]". Disponible en: http://unesco.org/delors/utopia.htm.

UNESCO. (1998), "Declaración Mundial sobre la Educación Superior en el Siglo XXI: Visión, Acción y Marco de Acción Prioritaria para el Cambio y el Desarrollo de la Educación Superior”. Disponible en : http://www.crue.org/dfunesco.htm.

WILSON, L y TAUBER, M. (1956). "The University Library: The Organization, Administration, And Functions Of Academic Libraries.", Columbia University.

ZURKOWSKI, P. (1974) "The Information Service Environment Relationships and Priori Ties", National Commission On Libraries And Information Science, Washington, D.F. 